\title{
Denaturation of proteins with beta-barrel topology induced by guanidine hydrochloride
}

\author{
Olesya V. Stepanenko a,* Irina M. Kuznetsova ${ }^{a}$, Vladislav V. Verkhusha ${ }^{\mathrm{b}}$, Maria Staiano $^{\mathrm{c}}$, \\ Sabato D'Auria ${ }^{c}$ and Konstantin K. Turoverov ${ }^{a}$ \\ ${ }^{a}$ Institute of Cytology of the Russian Academy of Sciences, St. Petersburg, Russia \\ ${ }^{\mathrm{b}}$ Albert Einstein College of Medicine, New York, USA \\ ${ }^{\mathrm{c}}$ Institute of Protein Biochemistry CNR, Napoli, Italy
}

\begin{abstract}
The stability of the representatives of two protein classes with $\beta$-barrel topology: porcine odorant-binding protein (OBP) and a number of fluorescent proteins (FPs), was studied. It was shown that both of them are significantly more stable than globular $\alpha$-helical and $\alpha / \beta$ proteins. At the same time the value of energy barrier between native and unfolded state for FPs exceeds that for OBP. It was found that the small guanidine hydrochloride concentrations induce local structural disturbances in proteins: changes in microenvironment of tryptophan residue in the case of odorant-binding protein and decrease in chromophore non-planarity in the case of green fluorescent protein.
\end{abstract}

Keywords: Odorant-binding proteins, fluorescent proteins, folding of proteins with beta-barrel topology, protein stability, small guanidine hydrochloride concentrations

\section{Introduction}

Protein folding is a matter of ongoing investigations in the field of molecular biology [5,13]. Folding of proteins with high content of $\beta$-structure and, especially, proteins with $\beta$-barrel topology is significantly less understood with respect to folding of $\alpha$-helical and $\alpha / \beta$ proteins. This work is focused on investigation of denaturation processes induced by guanidine hydrochloride $(\mathrm{GdnHCl})$ of proteins possessing $\beta$-barrel structure: porcine odorant-binding protein (OBP) and a number of fluorescent proteins (FPs).

Fluorescent proteins represent a large family of proteins with unique spectroscopic properties, i.e. the ability to absorb and in the most cases to emit visible light, arising from chromophore encapsulated in the rigid shell of protein [14]. The odorant-binding proteins are small extra-cellular proteins which are supposed to play a role in events of odorant molecules detection by carrying, deactivating and/or selecting odorant molecules $[7,16]$. In spite of the fact that both protein families belong to the architectural type of $\beta$-barrel, the folding pattern of these proteins has some differences. The $\beta$-barrel of FPs composed of $11 \beta$-strands is threaded by an $\alpha$-helix containing the chromophore formed from residues in the position 65-67 through an autocatalytic cyclization. The 8-stranded $\beta$-barrel of OBPs is flanked

\footnotetext{
${ }^{*}$ Corresponding author: Dr. Olesya V. Stepanenko, Institute of Cytology of the Russian Academy of Sciences, Tikhoretsky av. 4, 194064 St. Petersburg, Russia. Tel.: +7 81224719 57; Fax: +7 81224703 41; E-mail: lvs@ mail.cytspb.rssi.ru.
} 
by an $\alpha$-helix at the C-terminal end of the polypeptide chain. The $\beta$-barrel creates a central non-polar cavity whose role is to bind hydrophobic odorant molecules with dissociation constants ranging from nano-molar to micro-molar range.

Fluorescent proteins are of great importance for biological and medical research, being extensively used as a markers of gene expression and protein localization, as an indicator of protein-protein interactions and as a biosensor [15,21]. The odorant-binding proteins represent an exceptional interest because of investigation of olfaction mechanism and possibility to exploit these proteins as sensing probe of biosensors to different substances, including dangerous.

\section{Materials and methods}

\subsection{Materials}

Pig (Sus scrofa, breed Large White) nasal mucosa was obtained from the local slaughterhouse. The tissue was collected within 20 min after death and quickly utilized for the extraction of pOBP. Protein was purified from fresh porcine nasal tissue according to the procedure described by Dal Monte et al. [4]. This procedure yielded on average $15 \mathrm{mg}$ of purified pOBP-I from a single animal. Measurements were performed in $20 \mathrm{mM}$ TrisCl buffer, $\mathrm{pH}$ 7.5. Protein concentration was $0.1-0.5 \mathrm{mg} / \mathrm{ml}$.

The plasmids encoding EGFP, zFP506, mRFP1, "dimer2" and DsRed1 with polyhistidine tags were constructed as described previously [19] and were transformed into an Escherichia coli BL21(DE3) host (Invitrogen). The FP expression was induced by an incubation of the cells with $1 \mathrm{mM}$ IPTG (Nacalai tesque) during $24 \mathrm{~h}$ at $37^{\circ} \mathrm{C}$, and proteins were purified with Ni-NTA agarose (Qiagen). The purity of the recombinant proteins was not less than 95\%, as indicated by SDS-PAGE. Measurements were performed in $50 \mathrm{mM}$ TrisCl buffer, $\mathrm{pH}$ 8.0. Protein concentration was $0.05-0.5 \mathrm{mg} / \mathrm{ml}$.

$\mathrm{GdnHCl}$ (Nacalai Tesque, Japan) was used without further purification. The $\mathrm{GdnHCl}$ concentration was determined by refraction index with an Abbe refractometer (LOMO, Russia).

\subsection{Fluorescence measurements}

Fluorescence experiments were carried out using a homemade spectrofluorimeter with steady-state and time-resolved excitation [17]. The fluorescence of pOBP was excited at the long-wave absorption spectrum edge $(297 \mathrm{~nm})$, where the contribution of tyrosine residues in the bulk protein fluorescence is negligible, and at $280 \mathrm{~nm}$ where both tyrosine and tryptophan residues absorb. The position and form of the fluorescence spectra were characterized by the parameter $A=I_{320} / I_{365}$, where $I_{320}$ and $I_{365}$ are fluorescence intensities at $\lambda_{\mathrm{em}}=320$ and $365 \mathrm{~nm}$, respectively [18]. The values of parameter $A$ and of fluorescence spectrum were corrected by the instrument sensitivity. Specific "green" or "red" fluorescence of FPs was excited at $365 \mathrm{~nm}$, and emission was detected at $510 \mathrm{~nm}$ for EGFP and zFP506, at $584 \mathrm{~nm}$ for "dimer2", at $580 \mathrm{~nm}$ for DsRed1 and at $610 \mathrm{~nm}$ for mRFP1.

All kinetic experiments were performed in microcells 101.016-QS $5 \mathrm{~mm} \times 5 \mathrm{~mm}$ (Hellma, Germany). Unfolding of the protein was initiated by manual mixing of protein solution $(160 \mathrm{mkl})$ with buffer containing desired $\mathrm{GdnHCl}$ concentrations $(320 \mathrm{mkl})$. The dead time was determined from the control experiments to be about $4 \mathrm{~s}$. The spectrofluorimeter was equipped with thermostat that held a constant temperature of $25^{\circ} \mathrm{C}$ in the cell and in the special box where the solutions are held before mixing.

The equilibrium dependences of different fluorescent characteristics of pOBP on $\mathrm{GdnHCl}$ concentration were recorded after protein incubation in the solution of appropriate concentration at $4{ }^{\circ} \mathrm{C}$ overnight. 
Characteristic feature of the FP unfolding is a very low rate of their $\mathrm{N} \rightarrow \mathrm{U}$ transitions. Therefore, the shape of the denaturation curves depended significantly on the incubation time. Denaturation curves were characterized by the $C_{1 / 2}(t)$ values, which are the denaturant concentrations, corresponding to the middle of transition curves measured after the incubation of a protein in the presence of various denaturant concentrations for the time $t$. These data were used to plot $C_{1 / 2}$ versus incubation time dependencies (data is not present). Thermodynamic parameters were evaluated from the denaturation curves retrieved after the incubation of the corresponding solutions for time $t_{i}$, which corresponds to the incubation time at which the $C_{1 / 2}(t)$ curve reaches the plateau. In practice, the thermodynamic constants were calculated using the quasi-equilibrium dependencies obtained after the incubation of a given protein in the presence of various $\mathrm{GdnHCl}$ concentrations for 5 days. Protein stability in the absence of denaturant $\Delta G^{0}([0])$ was determined according to Nolting [11]. Approximation of denaturation curves of pOBP and FPs to a twostate model was performed via the nonlinear regression method using Sigma Plot program.

To analyze the fluorescence decay curves a special program was used [17,18]. The fitting routine was based on the non-linear least-squares method. Minimization was accomplished according to Marquardt [9]. P-terphenyl in ethanol and $\mathrm{N}$-acetyl-tryptophanamide in water were used as reference compounds [22].

\subsection{Circular dichroism measurements}

CD spectra were obtained with an Jasco-810 spectrophotometer (Jasco, Japan). Far UV CD spectra were recorded in a $1 \mathrm{~mm}$ path length cell, near UV CD spectra - in a $1.0 \mathrm{~cm}$ pathlength cell. For all spectra, an average of 5 scans was obtained. CD spectra of the appropriate buffers were recorded and subtracted from the protein spectra.

\subsection{Stern-Volmer quenching and estimation of the bimolecular quenching rates}

The conformational state of pOBP was further characterized by acrylamide-induced fluorescence quenching. Experiments were performed using excitation at $297 \mathrm{~nm}$ with fluorescence emission set at $360 \mathrm{~nm}$. Quenching data were plotted as the ratio of fluorescence in the absence of quencher $\left(I_{0}\right)$ to the intensity in the presence of quencher $(I)$ against quencher concentration. The resulting data were fit to dynamic parameters according to the Stern-Volmer equation $I_{0} / I=1+K_{\mathrm{SV}}[Q]$, where $K_{\mathrm{SV}}$ is the Stern-Volmer quenching constant and $[Q]$ the quencher concentration [6].

\section{Results and discussion}

\subsection{Denaturation of fluorescent proteins}

The list of studied here fluorescent proteins includes EGFP (green monomer [3]), zFP506 (green tetramer [10]), mRFP1 (red monomer [1]), "dimer2" (red dimer [1]) and DsRed1 (red tetramer [20]). All of them possess the $\beta$-barrel structure. The deconvolution of the far-UV CD spectra of FPs (and pOBP) using Provencher's algorithm [12] revealed about 3.5-5\% $\alpha$-helix, $41-44 \% \beta$-sheets and 20-23\% $\beta$-turns, which is consistent with crystallographic data. A unique characteristic feature of the fluorescent proteins unfolding is that this process is very slow. Kinetic analysis revealed that even at high $\mathrm{GdnHCl}$ concentration (5.6 M and higher) 50\%-reduction of the chromophore fluorescence intensity requires from several minutes (2.7 and 3.3 min for EGFP and "dimer2", correspondingly) to hours $(15,25$ and 

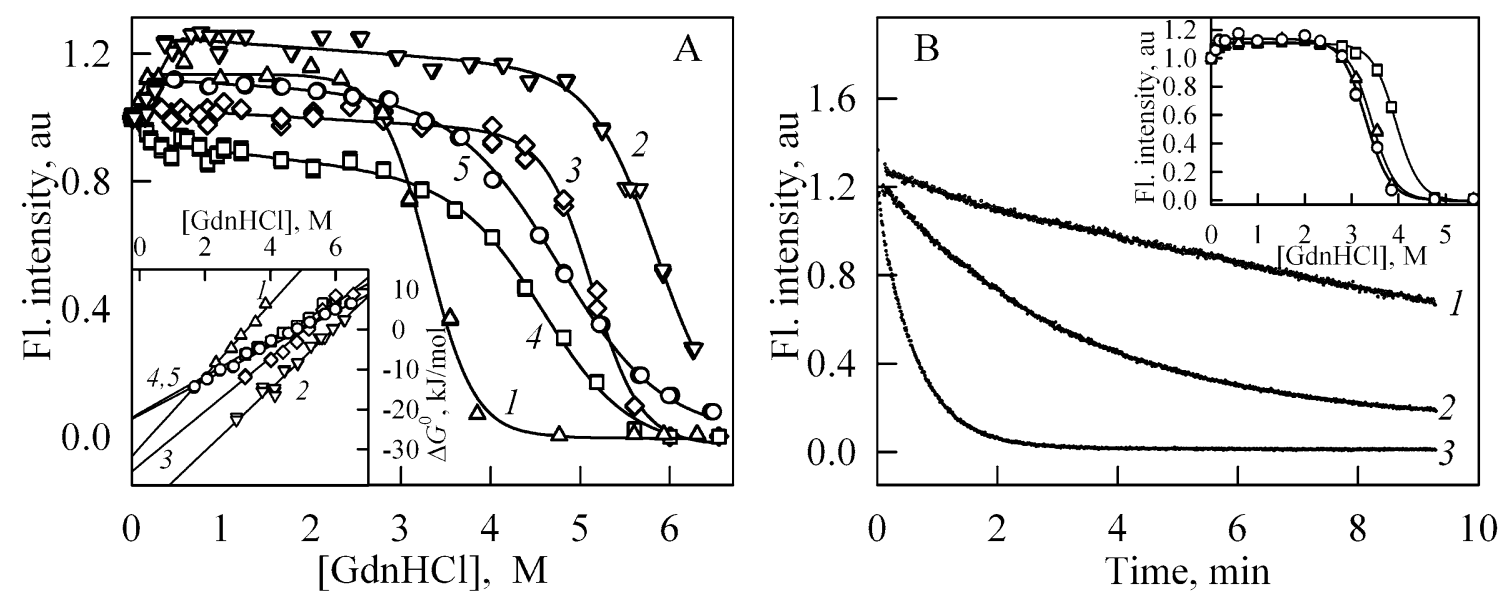

Fig. 1. Conformational stability of FPs. (A) GdnHCl-induced unfolding of EGFP (1, triangles), zFP506 (2, reversed triangles), mRFP1 (3, diamonds), "dimer2" (4, squares) and DsRed1 (5, circles) monitored by the changes of green or red fluorescence. Proteins have been incubated in the presence of the desired $\mathrm{GdnHCl}$ concentration for 5 days. Insert: the dependences of $\Delta G^{0}$ on the $\mathrm{GdnHCl}$ concentration for these proteins. The corresponding thermodynamics constants are given in Table 1. (B) Time dependence of fluorescence intensity of EGFP on unfolding in 4.0 (1), 5.6 (2) and $6.5 \mathrm{M} \mathrm{GdnHCl}(3)$. Kinetic curves have been normalized to the fluorescence intensity of native protein. Insert: the denaturation curves of green fluorescence for EGFP recorded after protein incubation in the presence of the desired $\mathrm{GdnHCl}$ concentration for 1 day (squares), 4 days (triangles) and 5 days (circles).

Table 1

Thermodynamic parameters of GdnHCl-induced unfolding of fluorescent proteins

\begin{tabular}{lccc}
\hline & $m\left(\mathrm{~kJ} \mathrm{~mol}^{-1} \mathrm{M}^{-1}\right)$ & $D_{50 \%}(\mathrm{M})$ & $\Delta G_{N-U}^{0}(0)\left(\mathrm{kJ} \mathrm{mol}^{-1}\right)$ \\
\hline EGFP & $9.0 \pm 0.7$ & 3.3 & $-29.5 \pm 2.0$ \\
zFP506 & $6.5 \pm 0.7$ & 5.9 & $-46.0 \pm 3.5$ \\
mRFP1 & $6.7 \pm 0.7$ & 5.0 & $-34.0 \pm 3.0$ \\
"dimer2" & $4.7 \pm 0.4$ & 4.6 & $-21.2 \pm 2.0$ \\
DsRed1 & $4.3 \pm 0.2$ & 4.8 & $-21.4 \pm 2.0$ \\
\hline
\end{tabular}

$67 \mathrm{~h}$ for mRFP1, DsRed1 and zFP506, respectively). For globular $\alpha$-helical proteins this value is in a range from several milliseconds to seconds. This means that native and unfolded states of fluorescent proteins are separated by high energy barrier.

The denaturation curves recorded after the incubation of the proteins in the presence of desired denaturant concentration for 5 days were analyzed with a two-state model (Fig. 1A). The results are shown in the insert to Fig. 1A and Table 1. These data revealed high stability of fluorescent proteins toward GdnHCl-induced unfolding.

Interesting effects were observed during investigation of EGFP denaturation. Firstly, the "green" fluorescence intensity recorded immediately after the mixing of EGFP with denaturant solution exceeds significantly the fluorescence level of a given protein in the absence of denaturant (Fig. 1B). Secondly, quasi-equilibrium curves also have shown that the addition of small $\mathrm{GdnHCl}$ concentrations $(0.1-0.2 \mathrm{M})$ to EGFP results in approximately $20 \%$ increase of "green" fluorescence intensity (Fig. 1B, insert). The addition of such denaturant concentrations has been shown to induce no noticeable changes of secondary structure as indicated by almost indistinguishable far UV CD spectra and just a small tertiary structure distortion recorded by negligible changes in near UV CD spectra (data is not shown). Previously it has 
been shown that the chromophore is slightly twisted in the native protein while it is planar in the unfolded protein $[2,8]$. It have been concluded that the effects observed in the small $\mathrm{GdnHCl}$ concentrations could be due to denaturant-induced removal of the protein scaffold tension. Under such conditions, the chromophore being inaccessible to the solvent becomes less "twisted" and, thus, more planar. This results in the increased conjugation of the chromophore $\pi$-bonds finally leading to the increase in the fluorescence quantum yield.

\subsection{Denaturation of odorant-binding proteins}

It is obviously that pOBP as well as fluorescent proteins posses high stability against GdnHCl-induced denaturation (Fig. 2B, insert). Indeed, the difference of protein free energy in the native and unfolded
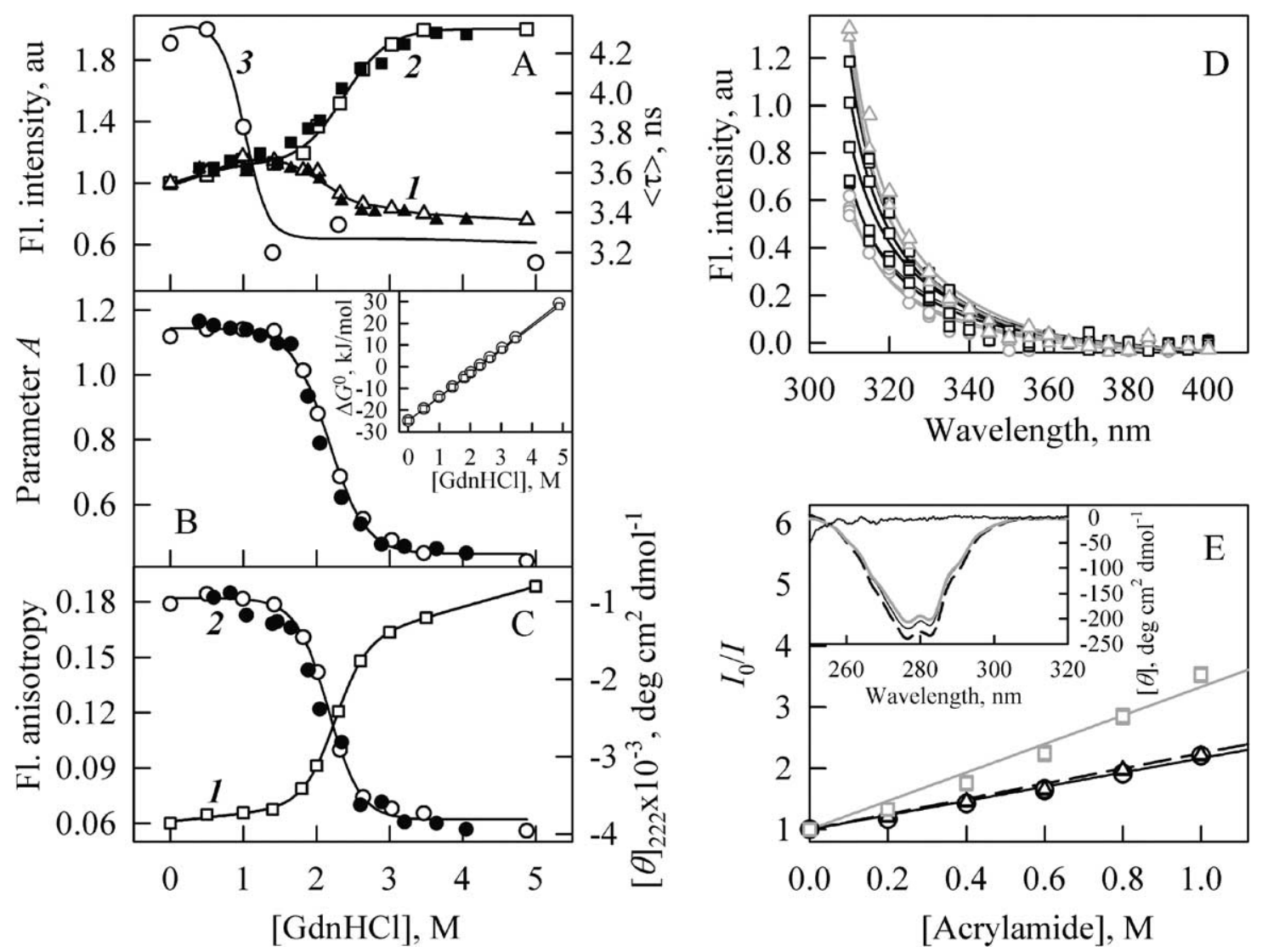

Fig. 2. GdnHCl-induced conformational changes of pOBP recorded by the changes of fluorescence intensity at $320 \mathrm{~nm}$ (curve 1 , A) and at $365 \mathrm{~nm}$ (curve 2, A), fluorescence lifetime (curve 3, A), parameter $A$ (B), ellipticity at $222 \mathrm{~nm}$ (curve 1, C) and fluorescence anisotropy at $365 \mathrm{~nm}$ (curve 2, C). Unfolding and refolding are presented by open and closed symbols, respectively. $\lambda_{\mathrm{ex}}=297 \mathrm{~nm}$. The dependences of $\Delta G^{0}$ on the $\mathrm{GdnHCl}$ concentration have been calculated on the base of the denaturation curves of parameter $A$ (squares, insert to panel $\mathrm{B}$ ) and ellipticity (circles, insert to panel $\mathrm{B}$ ). The conformational changes of pOBP are further characterized by the changes of the Tyr residues contribution to the bulk fluorescence of the protein (D) calculated as the difference between the fluorescence spectra excited at 280 and $297 \mathrm{~nm}$ (data corresponding to three subranges of $\mathrm{GdnHCl}$ concentrations, 0-1.4, 1.4-3.5 and 3.5-5.0 M, are depict as gray circles, black squares and gray triangles, respectively), acrylamide quenching of intrinsic fluorescence (E) and near-UV CD spectra (insert to panel E) in the presence of 0 (circles, solid line), 1.2 (triangles, dashed line) and $1.7 \mathrm{M} \mathrm{GdnHCl}$ (squares, gray line). 
states in the absence of denaturant is $-25 \mathrm{~kJ} / \mathrm{mol}$. At the same time, the rate of pOBP unfolding is higher as compared to fluorescent proteins, taking a few seconds at high denaturant concentration (data is not shown). In other words, the value of energy barrier between native and unfolded state for pOBP is essentially less than for fluorescent proteins.

Interestingly, the pre-denaturating $\mathrm{GdnHCl}$ concentrations resulted in increase of tryptophan fluorescence quantum yield and the decrease of fluorescence lifetime of the single tryptophan residue of pOBP, Trp16 (Fig. 2A). These effects are not accompanied by noticeable changes of protein secondary and tertiary structure which is manifested by invariance of parameter $A$, fluorescence anisotropy, the contribution of tyrosine residues to the bulk protein fluorescence, and far UV CD spectra (Fig. 2B-D). The accessibility of Trp16 to the solvent remains unchanged and near UV CD spectra become more pronounced (Fig. 2E), indicating the tryptophan microenvironment disturbances. This is likely leads to the disruption of the complex of Trp16 with the positively charged NZ atom of Lys 120, localized near Trp16 indole ring, and consequently to the enhancement of fluorescence intensity. The possibility of distance change between atom NZ of Lys 120 and the indole ring of the tryptophan residue of pOBP in water was confirmed by molecular dynamic simulations. At the same time, the increase of asymmetry of tryptophan residue microenvironment can also promote the formation of exciplexes of Trp16 with bound water molecules in close vicinity to indole ring and can explain the decrease of fluorescence lifetime. Thus, pre-denaturating $\mathrm{GdnHCl}$ concentrations induce local changes in microenvironment of Trp16 of pOBP.

\section{Acknowledgements}

This work was supported by NATO Grant CLG.983088 (MS and IMK), Contracts with FASI (02.740.11.5141) and FAE (P1198), Programs MCB RAS and "Leading Scientific School of RF" (1961.2008.4), Grants from SPb government MKN-185 (OVS, 2009) and NTD-3 (OVS, 2009) and Grant of the President of RF (MK-1181.2010.4).

\section{References}

[1] R.E. Campbell, O. Tour, A.E. Palmer, P.A. Steinbach, G.S. Baird, D.A. Zacharias and R.Y. Tsien, A monomeric red fluorescent protein, Proc. Natl. Acad. Sci. USA 99 (2002), 7877-7882.

[2] M.C. Chen, C.R. Lambert, J.D. Urgitis and M. Zimmer, Photoisomerization of green fluorescent protein and the dimensions of the chromophore cavity, Chem. Phys. 270 (2001), 157-164.

[3] B.P. Cormack, R.H. Valdivia and S. Falkow, FACSoptimized mutants of the green fluorescent protein (GFP), Gene 173 (1996), 33-38.

[4] M. Dal Monte, I. Andreini, R. Revoltella and P. Pelosi, Purification and characterization of two odorant-binding proteins from nasal tissue of rabbit and pig, Comp. Biochem. Physiol. B 99 (1991), 445-451.

[5] C.M. Dobson, Protein folding and misfolding, Nature 426 (2003), 884-890.

[6] M.R. Eftink, Fluorescence quenching: theory and applications, in: Topics in Fluorescence Spectroscopy, J.R. Lakowicz, ed., Plenum Press, New York, 1991, pp. 53-120.

[7] D.R. Flower, A.C. North and C.E. Sansom, The lipocalin protein family: structural and sequence overview, Biochim. Biophys. Acta 1482 (2000), 9-24.

[8] S.L. Maddalo and M. Zimmer, The role of the protein matrix in green fluorescent protein fluorescence, Photochem. Photobiol. 82 (2006), 367-372.

[9] D.W. Marquardt, An algorithm for least-squares estimation of nonlinear parameters, J. Soc. Indust. Appl. Math. 11 (1963), 431-441.

[10] M.V. Matz, A.F. Fradkov, Y.A. Labas, A.P. Savitsky, A.G. Zaraisky, M.L. Markelov and S.A. Lukyanov, Fluorescent proteins from nonbioluminescent Anthozoa species, Nat. Biotechnol. 17 (1999), 969-973. 
[11] B. Nolting, Protein Folding Kinetics: Biophysical Methods, Springer-Verlag, Berlin, 1999, p. 203.

[12] S.W. Provencher and J. Glockner, Estimation of globular protein secondary structure from circular dichroism, Biochemistry 20 (1981), 33-37.

[13] O.B. Ptitsyn, Molten globule and protein folding, Adv. Protein Chem. 47 (1995), 83-229.

[14] S.J. Remington, Fluorescent proteins: maturation, photochemistry and photophysics, Curr. Opin. Struct. Biol. 16 (2006), 714-721.

[15] O.V. Stepanenko, V.V. Verkhusha, I.M. Kuznetsova, V.N. Uversky and K.K. Turoverov, Fluorescent proteins as biomarkers and biosensors: throwing color lights on molecular and cellular processes, Curr. Protein Pept. Sci. 9 (2008), 338-369.

[16] M. Tegoni, P. Pelosi, F. Vincent, S. Spinelli, V. Campanacci, S. Grolli, R. Ramoni and C. Cambillau, Mammalian odorant binding proteins, Biochim. Biophys. Acta 1482 (2000), 229-240.

[17] K.K. Turoverov, A.G. Biktashev, A.V. Dorofeiuk and I.M. Kuznetsova, A complex of apparatus and programs for the measurement of spectral, polarization and kinetic characteristics of fluorescence in solution, Tsitologiia 40 (1998), 806817.

[18] K.K. Turoverov and I.M. Kuznetsova, Intrinsic fluorescence of actin, J. Fluoresc. 13 (2003), 41-57.

[19] V.V. Verkhusha, H. Otsuna, T. Awasaki, H. Oda, S. Tsukita and K. Ito, An enhanced mutant of red fluorescent protein DsRed for double labeling and developmental timer of neural fiber bundle formation, J. Biol. Chem. 276 (2001), 2962129624.

[20] D. Yarbrough, R.M. Wachter, K. Kallio, M.V. Matz and S.J. Remington, Refined crystal structure of DsRed, a red fluorescent protein from coral, at 2.0 Å resolution, Proc. Natl. Acad. Sci. USA 98 (2001), 462-467.

[21] M. Zimmer, Green fluorescent protein (GFP): applications, structure, and related photophysical behavior, Chem. Rev. 102 (2002), 759-781.

[22] M. Zuker, A.G. Szabo, L. Bramall, D.T. Krajcarski and B. Selinger, Deltafunction convolution method (DFCM) for fluorescence decay experiments, Rev. Sci. Instrum. 56 (1985), 14-22. 


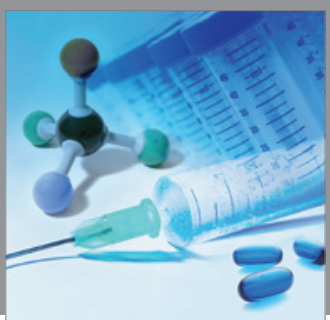

International Journal of

Medicinal Chemistry

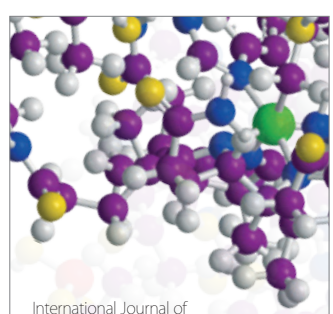

Carbohydrate Chemistry

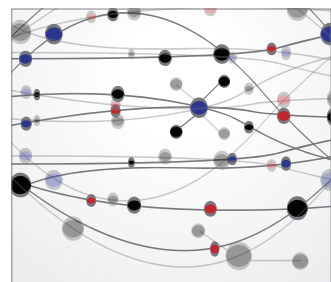

The Scientific World Journal
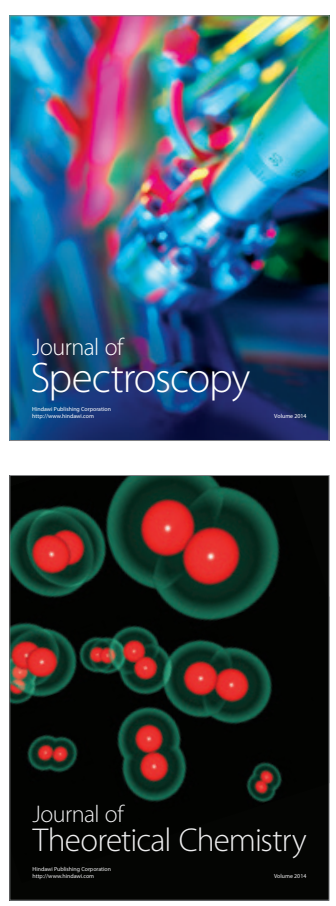
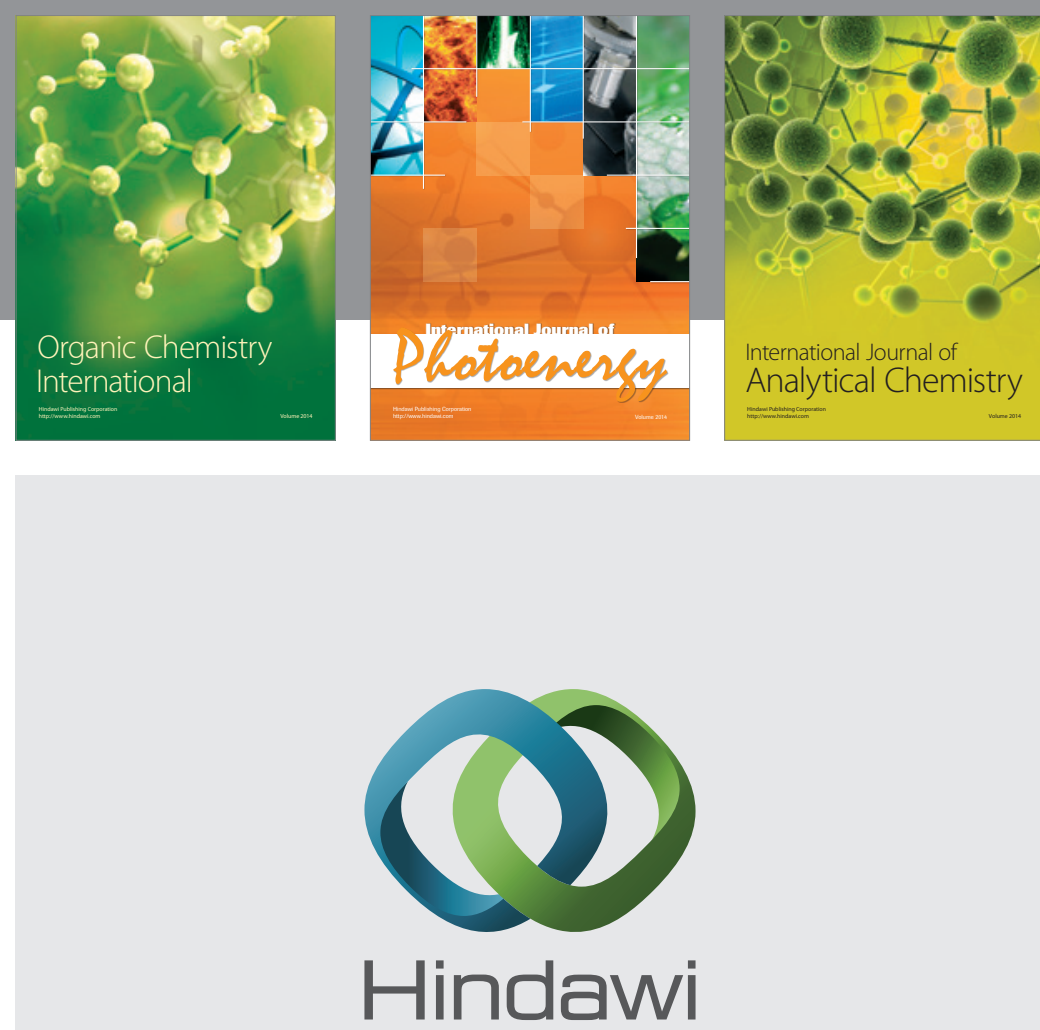

Submit your manuscripts at

http://www.hindawi.com
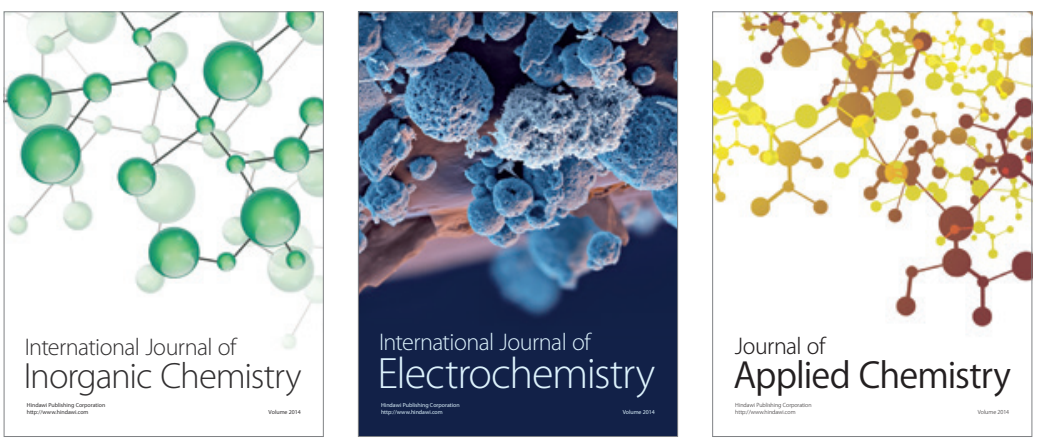

Journal of

Applied Chemistry
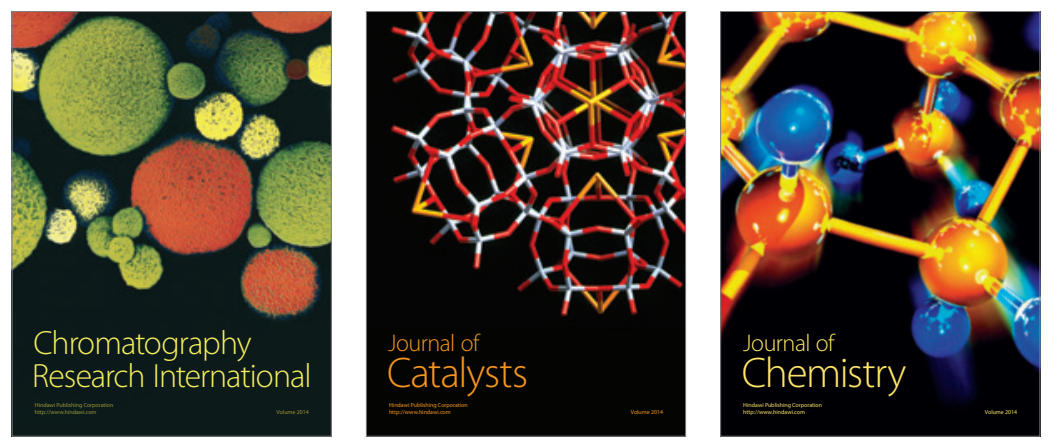
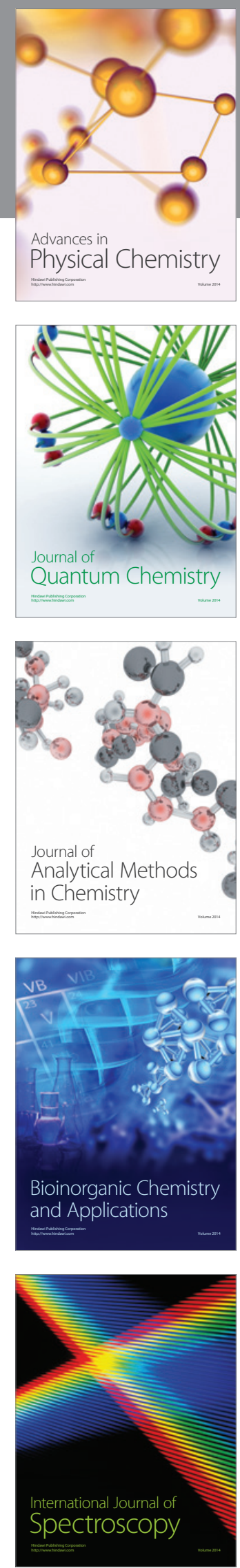